\title{
El Proyecto ACERTO: un protocolo multimodal barato y eficaz para América Latina
}

\author{
The ACERTO Project: an inexpensive and effective multimodal \\ protocol for Latin America \\ O projeto ACERTO: um protocolo multimodal económico e eficaz para a América Latina
}

José Eduardo de Aguilar-Nascimento ${ }^{1 *}$, Diana B. Dock-Nascimento², Jessika Cadavid Sierra ${ }^{3}$

Recibido: 4 de junio de 2019. Aceptado para publicación: 5 de agosto de 2019.

Publicado en línea: 9 de noviembre de 2019

https://doi.org/10.35454/rncm.v3n1.018

\begin{abstract}
Resumen
El proyecto ACERTO es un programa multimodal de cuidados perioperatorios. El conjunto de rutinas y prescripciones del proyecto ACERTO tiene como fundamento la medicina basada en evidencia en contraposición con cuidados tradicionales, muchas veces empíricos y enseñados en la rutina misma por generaciones sucesivas de cirujanos. Cuidados tradicionales como el uso de sonda nasogástrica, drenajes abdominales, preparación preoperatorio del colon, ayuno preoperatorio de 6 - 8 horas son modificados en el proyecto basado en la evidencia de estudios aleatorizados $y$ metaanálisis. Además de eso, el proyecto refuerza la restricción de fluidos intravenosos y el uso de terapia nutricional en el perioperatorio. La aplicación del proyecto ACERTO desde 2005 ha modificado resultados, mostrando reducción significativa de días de internación y morbilidad postoperatoria en sucesivos estudios clínicos. El proyecto ACERTO es de uso gratuito.
\end{abstract}

Palabras clave: cuidados perioperatorios, ayuno preoperatorio, terapia nutricional, complicaciones postoperatorias, hidratación venosa, preparación mecánica del colon.

\section{Summary}

The ACERTO project is a multimodal peri-operative care program. The set of routines and prescriptions of the ACERTO project is based on evidence-based medicine as opposed to traditional care, often empirical and taught in the same routine by successive generations of surgeons. Traditional care such as the use of nasogastric tube, abdominal drainage, preoperative colonic preparation, preoperative fasting of 6-8 hours are modified in the project based on evidence from randomized studies and meta-analysis. In addition, the project reinforces the restriction of intravenous fluids and the use of nutritional therapy in the peri-operative. The application of the ACERTO project since 2005 has been modifying results, showing significant reduction of hospitalization days and postoperative morbidity in successive clinical studies. The ACERTO project is free to use.

Keywords: Peri-operative care; Preoperative fasting; Nutritional therapy; Postoperative complications; Intravenous fluids; Preoperative colonic preparation.

\section{Resumo}

$O$ projeto ACERTO é um programa multimodal de cuidados peri-operatórios. $\mathrm{O}$ conjunto de rotinas e prescrições do projeto ACERTO tem como base a medicina baseada em evidência em contraposição a cuidados tradicionais, muitas vezes empíricos e, ensinado a beira do leito, por gerações sucessivas de cirurgiões. Cuidados tradicionais como uso de sonda nasogástrica, drenos abdominais, preparo préoperatório do cólon, jejum pré-operatório de 6-8 horas são modificados no projeto com base na evidência de estudos randomizados e meta-analises. Alem disso, o projeto reforça a restrição de fluidos intravenosos e o uso de terapia nutricional no peri-operatório. A aplicação do projeto ACERTO desde 2005 modificou resultados com queda significante de dias de internação e morbidade pós-operatória em sucessivos estudos clínicos. O projeto ACERTO é de uso gratuito.

Palavras-chave: cuidados peri-operatórios, jejum pré-operatório, terapia nutricional, complicações pos-operatórias, hidratação venosa, preparo mecânico de cólon.
Faculdade de Medicina da Universidade Federal de Mato Grosso, UFMT; Director del curso de Medicina - UNIVAG, Brasil.

2 Faculdade de Medicina da Universidade Federal de Mato Grosso, UFMT, Brasil. 3 Universidad Federal de Mato Grosso (UFMT), Brasil; Nutricionista Dietista
de la Universidad de Antioquia (UDEA), Colombia.

*Correspondencia: José Eduardo de Aguilar Nascimento je.nascimentocba@gmail.com 


\section{INTRODUCCIÓN}

En los últimos años, varios estudios se han preocupado por la recuperación del paciente quirúrgico en términos de resultados (endpoints) importantes, tales como tiempo de internación, morbilidad y mortalidad postoperatoria. La explicación para la llegada de estos estudios, en parte, es el desarrollo de estrategias que minimicen la respuesta orgánica al trauma, mejoren la calidad de la atención y aceleren la recuperación del paciente ${ }^{(1,2)}$.

Programas multimodales ("fast track") hacen referencia al conjunto de intervenciones que pretenden facilitar y acelerar la recuperación postoperatoria a través de prescripciones y cuidados modernos. Esas nuevas rutinas de cuidados, generalmente siguen el nuevo paradigma de la medicina basada en evidencia. En esa nueva visión, cuidados tradicionales, muchas veces empíricos y transmitidos por generaciones de cirujanos, en la rutina misma, se contraponen a otras rutinas basadas en estudios aleatorizados, controlados y en metaanálisis. Algunas enseñanzas de ese tipo, tales como tiempo de ayuno preoperatorio, realimentación postoperatoria, reposo en cama, preparación mecánica del colon, uso rutinario de sonda nasogástrica y drenajes, han sido mostrados en estudios aleatorizados y controlados como inútiles y muchas veces peligrosos ${ }^{(3)}$.

\section{CONCEPTO DE PROTOCOLO MULTIMODAL O FAST TRACK}

El grupo ERAS (Enhanced Recovery After Surgery), compuesto por médicos, especialmente cirujanos colorrectales de algunos países del norte de Europa, publicó en 2005 un interesante consenso sobre cuidados perio- peratorios $^{(4)}$, en el que presentaron varias modificaciones a cuidados nutricionales, basados en estudios controlados, aleatorizados y en metaanálisis. Las principales modificaciones presentadas por el grupo ERAS fueron adaptadas a la realidad nacional por el proyecto ACERTO y pueden ser observadas en la Figura 1.

Uno los objetivos de los programas multimodales es la disminución del estrés quirúrgico a través de la reducción de la respuesta orgánica al trauma. En ese contexto, la aparición de la videolaparoscopia, indiscutiblemente, trajo beneficios al cirujano y al paciente, de tal manera que muchos cirujanos que aprendieron de la manera tradicional pasaron a abreviar la internación del paciente y no utilizar de rutina cuidados perioperatorios tradicionales, tales como sonda nasogástrica y catéter urinario. La Tabla 1 sintetiza varios principios que pueden minimizar la respuesta orgánica al trauma. La Figura 2 muestra las principales diferencias entre un protocolo de cuidados perioperatorios tradicionales y un multimodal.

Tabla 1. Principios para la reducción de la respuesta orgánica al trauma ${ }^{(5)}$

\begin{tabular}{|l|}
\hline Cirugía mínimamente invasiva \\
\hline Prevención de hipotermia perioperatoria \\
\hline Bebida con carbohidratos 2 horas antes de la operación \\
\hline Nutrición perioperatoria \\
\hline Optimización del uso de fluidos intravenosos \\
\hline Analgesia con fármacos no opioides \\
\hline Uso de procinéticos \\
\hline Anestesia mixta (general + bloqueo regional) \\
\hline
\end{tabular}

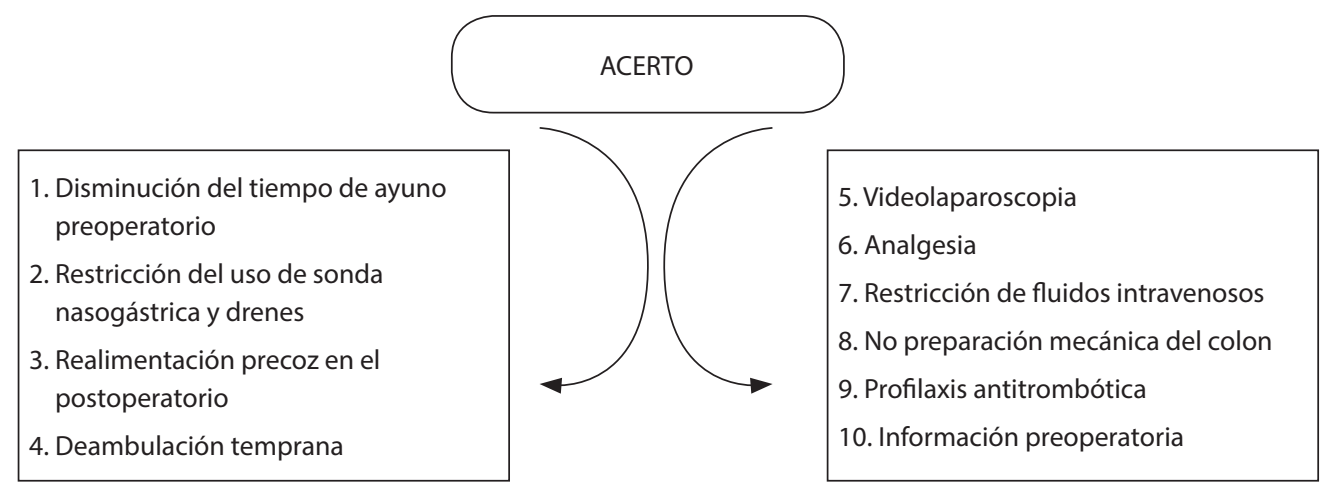

Figura 1. Componentes del programa multimodal de cuidado perioperatorio - ACERTO (Aceleración de la Recuperación Total Postoperatoria) $)^{(5)}$. 


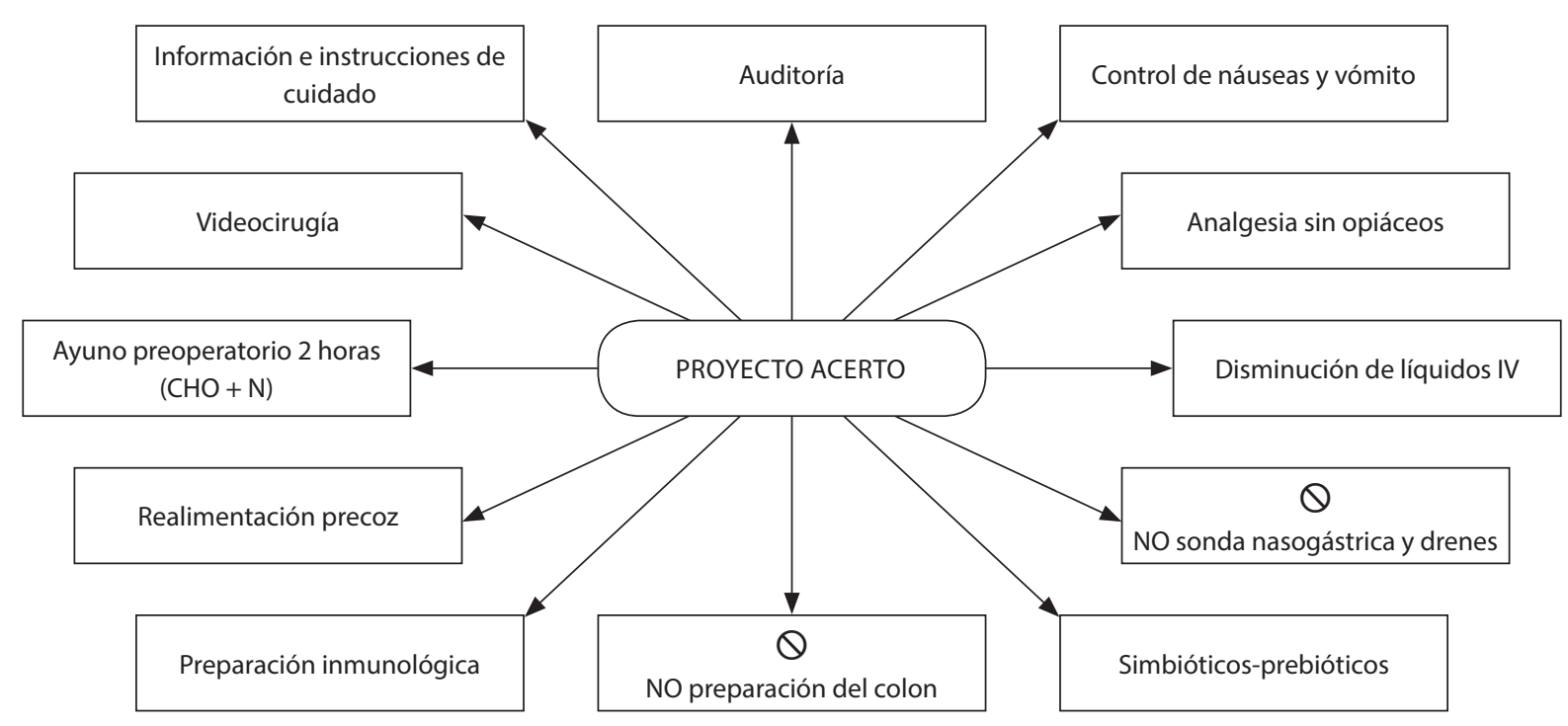

Figura 2. Elementos del protocolo ACERTO.

Los resultados iniciales con el uso de protocolos multimodales estuvieron basados en estudios no aleatorizados de observación. En la actualidad, existen muchos estudios aleatorizados y metaanálisis que muestran la consistencia de esos programas en derivar mejores desenlaces, especialmente en cirugías colorrectales ${ }^{(5,6)}$. Estos resultados muestran que hay reducción significativa de morbilidad y de costos hospitalarios gracias a la adopción de esos programas. Wind et al. en revisión sistemática de seis estudios aleatorizados y 512 pacientes, mostraron que pacientes sometidos a operaciones de intestino grueso por protocolos fast-track permanecieron hospitalizados 1,5 días menos (- 1,56 días, IC $95 \%$ : 2,61 - 0,50 días) y hubo disminución de la morbilidad postoperatoria en aproximadamente $50 \%(R R=0,54$, IC $95 \%: 0,42-0,69)^{(5)}$.

\section{EL PROTOCOLO ACERTO}

El número de operaciones realizadas en el mundo es muy grande. En los Estados Unidos, se realizan cerca de 30 millones de procedimientos cada año, consumiendo una gran cantidad de recursos de salud. En Brasil, en 2017 ocurrieron 11.338.039 internaciones en el país, 3.123.517 debido a procedimientos quirúrgicos (27,5\%), con un costo promedio de R\$ 1184,32 (Reales) por internación ${ }^{(7)}$. Esos datos demuestran que, en términos nacionales, consideraciones que implican costos y resultados en procedimientos quirúrgicos son realmente importantes $y$, en consecuencia, debe haber una preocupación de los servicios de salud con gastos y mejora de resultados en la atención en ese grupo de pacientes. En Brasil, se optó por un protocolo, sin costos adicionales de pagos de honorarios, para plataformas que funcionan en algunos lugares del mundo (más acorde con gastos en Salud Pública en Brasil y posiblemente en América Latina).

Un punto crucial para la implementación es el conocimiento epidemiológico local de los resultados. El número de procedimientos realizados por mes y año, la tasa de morbilidad y el tiempo de internación postoperatorio de un determinado hospital, por ejemplo, no pueden estar basados en presunción. Un buen sistema debe auditar algunos parámetros en pacientes internados. Conocer el promedio de horas de ayuno preoperatorio o el porcentaje de uso rutinario de sonda nasogástrica es interesante. Las auditorías deben demostrar los resultados del servicio y del hospital, señalar las principales operaciones y preferencias del equipo. Esa información tiene que ser divulgada en reuniones de servicio, gráficos en el centro quirúrgico, boletín del hospital, e-mails, etc. Imaginario y realidad deben ser confrontados y las discusiones ocurrir a partir de datos reales, deben producir cambios basados en hechos y no en presunciones. Para ejemplificar este punto importante, una auditoría en nuestro servicio realizada con el objetivo de evaluar la cantidad real de fluidos infundidos por vía intravenosa en pacientes sometidos a operaciones de gran tamaño, mostró que entre el primero y el cuarto día postoperatorio se 
infundieron 12,8 L $(6,4 \mathrm{~L}-17,5 \mathrm{~L})$ de soluciones cristaloides. De ese total, 9,5 litros $(74,3 \%)$ correspondieron a fluidos prescritos y $3,3 \mathrm{~L}(25,7 \%)$ a diluyentes y medicaciones venosas. Se concluyó que la prescripción médica no contiene el volumen real de fluidos cristaloides intravenosos infundido. El volumen de diluyentes y medicamentos intravenosos puede llegar a $25 \%$ de la carga hídrica prescrita ${ }^{(8)}$.

Dentro del protocolo ACERTO (Tabla 2), el paciente inmediatamente después de la internación recibe información importante sobre su operación y se le instruye acerca de la forma de colaborar para un rápido retorno al hogar. La evaluación del riesgo nutricional y la implementación de la INTERNUTI (Intervención Nutricional Inmediata), si son necesarias, forman parte del preoperatorio. El diagrama de flujo de la INTERNUTI puede ser observado en la Figura 3.

En la rutina del protocolo ACERTO, el alta médica está condicionada por 4 factores: 1 . el paciente debe estar recibiendo dieta y sin infusión intravenosa, 2. el dolor debe estar ausente o controlado con analgésicos orales, 3. el paciente debe estar deambulando solo, o con poca ayuda, y 4. el paciente debe desear el alta médica. El protocolo ACERTO fue recientemente adoptado por el Colegio Brasileño de Cirujanos y por la Sociedad Brasileña de Nutrición Parenteral y Enteral como directriz de cuidados $^{(9)}$.

\section{DISMINUCIÓN DEL TIEMPO DE AYUNO PREOPERATORIO}

El ayuno nocturno preoperatorio fue instituido cuando las técnicas anestésicas aún eran rudimentarias para pre- venir complicaciones pulmonares asociadas a vómitos y aspiraciones del contenido gástrico. La razón de esa rutina es garantizar el vaciamiento gástrico y evitar la broncoaspiración en el momento de la inducción anestésica. La revisión de libros de texto del siglo pasado muestra que el dogma del ayuno preoperatorio de 8 - 12 horas fue establecido a partir del relato de casos de aspiración bronco-pulmonar en situaciones cuya inducción anestésica se dio en operaciones de urgencia y emergencia. A principios del siglo XIX, los pacientes tenían permiso de ingerir una pequeña taza de té pocas horas antes de la operación. Sin embargo, la conducta que se popularizó fue la de aplicar ayuno ("nada por la boca" o "nada vía oral"), a partir de la medianoche para pacientes que tuvieran su operación programada para el período matutino, y el permiso de ingerir algún alimento leve (té y galletas) para pacientes que fueran a ser operados en la tarde ${ }^{(10)}$. Sin embargo, las cirugías se retrasan y el ayuno preoperatorio aumenta hasta llegar en promedio a 18 horas $^{(11)}$. En la Tabla 3 se presentan las principales razones del retraso de las cirugías.

En varias circunstancias, el imaginario de una prescripción no es efectivamente cumplido. En el período anterior a la implantación del Proyecto ACERTO, en el Hospital Universitario Julio Muller (FCM-UFMT), el tiempo preconizado para ayuno preoperatorio era de ocho horas. Una auditoría realizada en la implantación del protocolo ACERTO mostró claramente que el tiempo de ayuno preconizado y prescrito en el preoperatorio de cirugías electivas era mucho mayor llegando a las 16 horas en promedio ${ }^{(12)}$. Incluso después de la implantación del ayuno de 2 horas, ese tiempo en promedio fue mayor (4 horas). Las razones para esa dila-

Tabla 2. Comparación entre el protocolo convencional y el ACERTO en un paciente candidato a una resección de colon con anastomosis primaria ${ }^{(5)}$

\begin{tabular}{|l|l|}
\hline \multicolumn{1}{|c|}{ Convencional } & \multicolumn{1}{c|}{ ACERTO } \\
\hline Sin terapia nutricional (TN) preoperatoria & TN preoperatoria 7 - 14 días \\
\hline Poca o ninguna explicación sobre el perioperatorio & Información sobre el perioperatorio \\
\hline Preparación del colon & NO preparación mecánica del colon \\
\hline Sonda nasogástrica y drenes & NO sondas y drenes \\
\hline Ayuno de 8 horas & Ayuno de 2 horas (carbohidratos) \\
\hline Realimentación en el $2^{\circ}-4^{\circ}$ día & Realimentación en el PO inmediato o $1^{\circ}$ día \\
\hline Fluidos IV hasta el $4^{\circ}-7^{\circ}$ día & Fluidos hasta el $1^{\circ}$ día \\
\hline
\end{tabular}




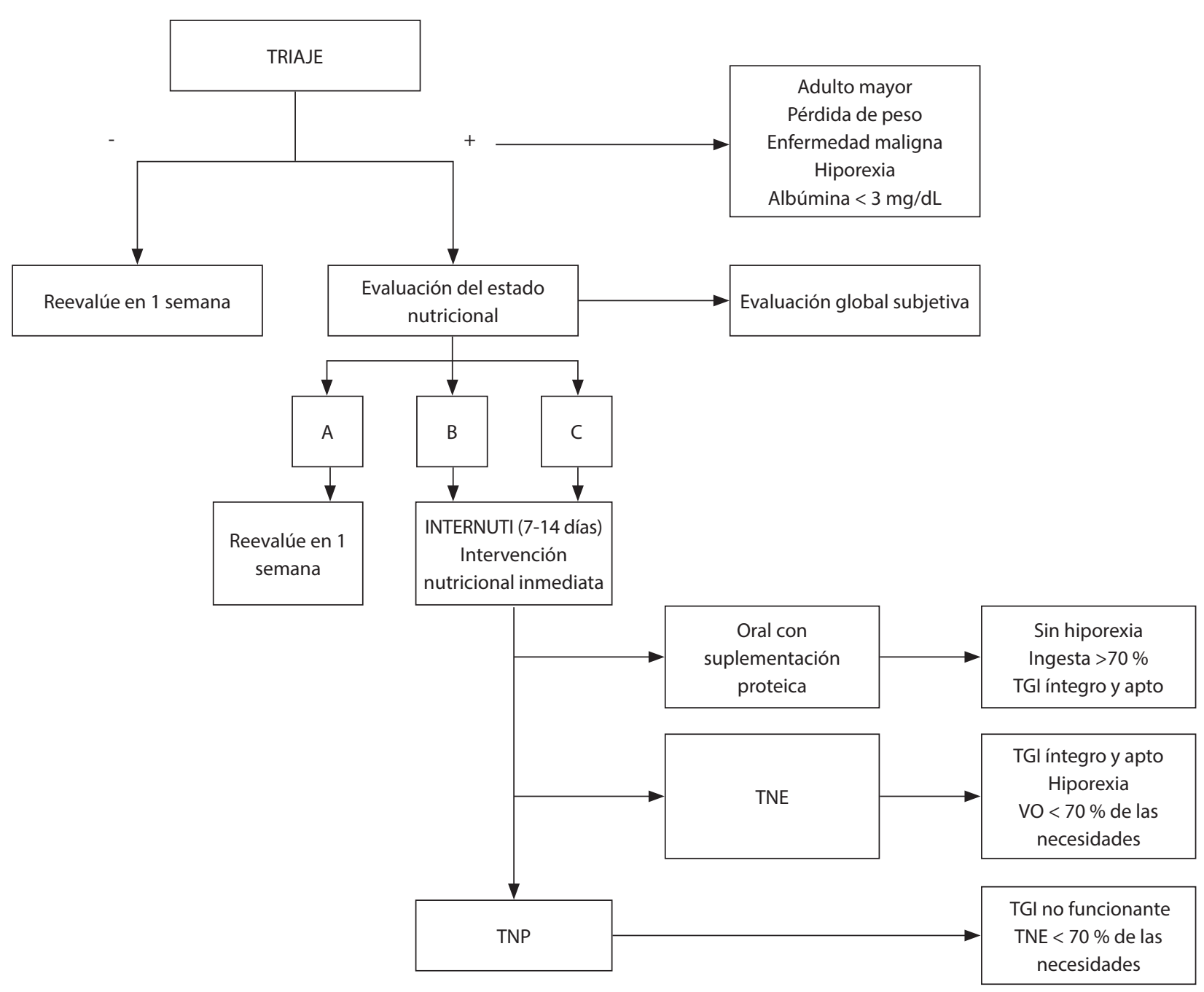

Figura 3. Flujograma de intervención nutricional inmediata (INTERNUTI) en el paciente quirúrgico candidato a operaciones de mediana y alta complejidad(5).

Tabla 3. Razones que prolongan el tiempo de ayuno preoperatorio prescrito ${ }^{(5)}$

\begin{tabular}{|c|}
\hline 1. Retraso del cirujano o del anestesiólogo \\
\hline $\begin{array}{l}\text { 2. Retraso en la burocracia (gestión administrativa) de } \\
\text { internación del paciente }\end{array}$ \\
\hline 3. Retraso en el inicio de la cirugía del primer turno \\
\hline $\begin{array}{l}\text { 4. "Adherencia" del paciente a la recomendación de ayuno } \\
\text { preoperatorio }\end{array}$ \\
\hline 5. Inducción anestésica prolongada \\
\hline $\begin{array}{l}\text { 6. Reprogramación de la operación para el segundo o tercer } \\
\text { turno quirúrgico }\end{array}$ \\
\hline 7. Reprogramación de la operación para otro período del día \\
\hline
\end{tabular}

tación del período de ayuno preoperatorio se deben al retraso de las operaciones, el cambio de horario de las mismas y el aumento del tiempo de ayuno por parte del propio paciente.

La posibilidad de uso de líquidos claros, hasta dos horas antes de la operación, abrió precedentes para que algunos autores estudiaran la posibilidad de que tales soluciones pudieran ser enriquecidas con sustratos energéticos de rápida absorción y que no interfirieran con el vaciamiento gástrico. Diferentes trabajos publicados demostraron que el uso de una solución líquida enriquecida con carbohidratos determinaba mayor satisfacción, menor irritabilidad, aumento del $\mathrm{pH}$ gástrico y, especialmente, reducía la respuesta catabólica al 
estrés quirúrgico, con consecuente mejora de la recuperación postoperatoria ${ }^{(4,12)}$.

Como fundamentación básica para este cambio de paradigma en la prescripción de ayuno preoperatorio, es importante considerar que la respuesta orgánica al trauma quirúrgico puede ser incrementada por la respuesta metabólica al ayuno preoperatorio prolongado. En conjunto, estos factores contribuirán en forma relevante a una respuesta orgánica aumentada ${ }^{(13)}$. Después de algunas horas de ayuno, se instala una resistencia periférica a la insulina y los índices de insulina aumentan por feed-back. Faria et al. lo demostraron claramente en un estudio aleatorizado reciente ${ }^{(14)}$. Además, cuanto mayor es la resistencia a la insulina, mayor es el tiempo de internación ${ }^{(15)}$.

Una revisión de la Cochrane ${ }^{(16)}$ identificó y analizó las evidencias más fuertes en relación con la disminución del tiempo de ayuno preoperatorio y su relación con complicaciones. Fueron incluidos 22 estudios y un total de 2.270 participantes. No hubo caso de aspiración por abreviación del ayuno para 2 horas con bebida de carbohidratos. De manera consistente, varias guías de manejo de sociedades científicas de anestesiólogos, publicados antes del estudio de la Cochrane, ya defendían la indicación de líquidos claros hasta dos horas antes de la operación quirúrgica (ASA 1999; NNCG 1993; AAGBI 2001) y hasta $150 \mathrm{~mL}$ de líquidos claros inclusive una hora antes de la operación con medicamentos orales (NNCG 1993).

En los últimos años hubo un desarrollo de nuevas bebidas para la reducción del ayuno preoperatorio. Bebidas que contienen solo maltodextrina, soluciones con la adición de proteínas, aminoácidos, antioxidantes, electrolitos, oligo-elementos y vitaminas fueron estudiadas, y están disponibles en el mercado. La adición de proteínas o aminoácidos mejora aún más la resistencia insulínica, disminuye la pérdida de masa magra y, por lo tanto, disminuye aún más la respuesta orgánica al trauma. La seguridad del vaciamiento gástrico con una bebida que contenga carbohidratos asociada a glutamina o proteína hidrolizada ocurrió en al menos cuatro estudios aleatorizados ${ }^{(17,18)}$.

\section{RESTRICCIÓN DE FLUIDOS INTRAVENOSOS}

Líquidos y electrolitos son infundidos con la finalidad de reponer las pérdidas ocasionadas durante el acto operatorio y también para mantener la homeostasis durante el período de tiempo en que la ingesta oral es imposible. Estas necesidades se relacionan de manera directa con el peso del paciente, el tamaño y la duración del acto operatorio. Tradicionalmente, el cálculo se realiza basado en una fórmula empírica, a menudo favoreciendo una reposición generosa ${ }^{(19)}$. Con esa conducta convencional, pacientes sometidos a operaciones colorrectales reciben en promedio 3,5 - 5 litros de fluido intravenoso el día de la operación y se mantienen en los primeros días de recuperación quirúrgica (3 - 4 días) con hidratación venosa, muchas veces con "suero fisiológico ", recibiendo 2 - 3 litros / día. Esta reposición, asociada a la dificultad de excreción fisiológica de sodio, de cloro y de agua durante ese período, como respuesta fisiológica a la agresión, determina una retención hídrica importante, con ganancia de peso corporal en torno a 3 - 6 kilos en el período postoperatorio ${ }^{(20,21)}$.

Se ha demostrado que esa sobrecarga causa edema generalizado de tejidos, con innumerables consecuencias clínicas. Hay una alteración de la función cardiopulmonar con consecuente disminución de la oxigenación sanguínea y deletéreas repercusiones en todo el organismo. Particularmente, en el tubo digestivo hay edema esplácnico, aumento de la presión intraabdominal, disminución del flujo mesentérico con mantenimiento del íleo paralítico, aumento de la permeabilidad de la mucosa y perjuicio del proceso cicatricial.

En un estudio multicéntrico, Brandstrup et al. ${ }^{(20)}$ compararon dos regímenes de reposición perioperatoria de líquidos. Se concluyó que en el grupo restringido hubo reducción significativa de complicaciones postoperatorias (33\% vs $51 \%$ ); cardiopulmonar (7\% vs $24 \%$ ) y relacionadas con la cicatrización ( $16 \%$ vs $31 \%$ ); destacando no haber observado ningún posible efecto adverso en el régimen de restricción (a pesar de que la restricción de fluidos implicó menor débito urinario, ningún paciente evolucionó con insuficiencia renal). En 2009, se presentó un consenso en el Reino Unido, el GIFTASUP (British Consensus Guidelines on Intravenous Fluid Therapy for Adult Surgical Patients), que determina conductas para la hidratación perioperatoria ${ }^{(21)}$. Según el consenso, la hidratación restrictiva en cirugías abdominales de gran tamaño recibe la clasificación IA de evidencia, para cirugías ortopédicas de gran tamaño la clasificación IB y el soporte para optimización del débito cardíaco en hidratación restrictiva con el uso de drogas vasoactivas, IB. Es claro que la tendencia de restricción hídrica ha sido confirmada con mayor fuerza, y datos actuales más determinantes dentro de la evidencia científica. 


\section{REALIMENTACIÓN PRECOZ EN EL POSTOPERATORIO}

La adopción del ayuno después de operaciones con manipulación de la cavidad abdominal y, en especial, después de la realización de anastomosis colorrectales, se enseña a residentes de cirugía desde hace mucho tiempo. Por tradición, el retorno de la dieta a los pacientes sometidos a anastomosis intestinal ha sido prescrito solo después del regreso del peristaltismo, caracterizado clínicamente por la aparición de los ruidos hidroaéreos y la eliminación de gases ${ }^{(22)}$. Con ello, el ayuno postoperatorio se prolonga por un período de 2 a 5 días y, durante ese período, el paciente queda, generalmente, recibiendo solo hidratación venosa con soluciones cristalinas, con un mínimo de calorías y sin suministro de nitrógeno. Eso puede provocar un volumen exagerado de fluidos que puede llegar a $10 \mathrm{~L}$ en 3 días y provocar una ganancia inadecuada de peso, náuseas, vómitos y mayor tiempo de íleo.

Sin embargo, ese tipo de conducta ha sido discutida y contrarrestada por la literatura reciente. En realidad, varios trabajos controlados y aleatorizados ${ }^{(23,24)}$ y metaanálisis $^{(25)}$ demuestran que la realimentación precoz después de operaciones, involucrando resecciones y anastomosis intestinales, puede ser conducida sin riesgos y con beneficios potenciales en los pacientes como: alta más precoz, menor incidencia de complicaciones infecciosas y disminución de $\operatorname{costos}^{(26)}$. El uso de sonda nasogástrica como rutina tampoco tiene soporte en la evidencia y, en realidad, hasta puede complicar el postoperatorio.

Estudios sobre la fisiología de la motilidad del tubo digestivo han demostrado que el retorno al peristaltismo normal en el postoperatorio es más rápido en el intestino delgado, que retorna a su función 4 a 8 horas después de la operación. El colon izquierdo y el estómago regresan más lento, con promedio de 24 horas, aproximadamente. Sin embargo, las contracciones en la región antral del estómago regresan a la normalidad dos horas después del acto operatorio y, en realidad, pueden estar exacerbadas en las primeras veinte horas del postoperatorio ${ }^{(27)}$. Así, desde el punto de vista de la motilidad, es posible también la reintroducción precoz de la dieta en el postoperatorio de cirugías realizadas en el tubo digestivo.

Aguilar-Nascimento y Gloetzer ${ }^{(23)}$ mostraron, en la enfermería del Hospital Universitario Julio Muller, que es posible realimentar a pacientes tras anastomosis colónicas sin riesgo y con disminución del tiempo de íleo y de permanencia hospitalaria. En metaanálisis,
Lewis et al ${ }^{(25)}$ pusieron fin a la antigua idea de los "riesgos" de una realimentación precoz en el postoperatorio con anastomosis intestinal.

Por lo tanto, no hay ninguna evidencia para dejar pacientes en ayuno por dos o más días en el postoperatorio. Por el contrario, estudios aleatorizados y controlados mostraron de manera repetida que la realimentación el mismo día de la operación o al día siguiente es segura y confiere mejores resultados. Realimentar precozmente al paciente disminuye el tiempo de internación, acelera la recuperación y es uno de los pilares del proyecto ACERTO.

\section{PREPARACIÓN MECÁNICA DEL COLON}

Una investigación realizada entre 808 cirujanos americanos, especialistas en cirugía colorrectal, realizada en 1997, apuntó que $100 \%$ de ellos indicaban esa rutina preoperatoria $^{(28)}$. La constatación de ese hecho mostró un gran desacuerdo entre la evidencia y la práctica ${ }^{(29)}$. Sin embargo, un estudio más reciente publicado en 2010 indicó un cambio de actitud por parte de cirujanos colorrectales en el Reino Unido e Irlanda. Ese estudio mostró que para la operación del colon derecho y resección abdomino-perineal del recto, pocos cirujanos continúan prescribiendo la preparación y que para el colon izquierdo la mayoría dispensa esa prescripción ${ }^{(30)}$.

La preparación mecánica del colon se estructura en la idea de retirar las heces del intestino grueso, ya que la población bacteriana en las heces es muy grande (del orden de $10^{14}$ bacterias por gramo de heces), y por lo tanto, gran carga contaminante. Sin embargo, los inconvenientes de la preparación mecánica del colon, tales como la incomodidad, la deshidratación y la necesidad de reposición hídrica por vía intravenosa, siempre se consideraron necesarios, teniendo en cuenta que el objetivo mayor era la minimización de la morbi-mortalidad.

Varios trabajos aleatorizados de la era de la medicina basada en evidencia y algunos metaanálisis ${ }^{(31-33)}$ han mostrado en forma consistente que la preparación mecánica preoperatoria del colon es prescindible. Una revisión sistemática incluyendo aproximadamente 5.000 casos mostró mayor incidencia de infección en el sitio quirúrgico con el uso de la preparación de colon $(\mathrm{OR}=1,40(1,05-1,87), \mathrm{p}=0,02)^{(34)}$.

\section{CONCLUSIÓN}

En cirugía general, la técnica operativa mejorada es esencial. Sin embargo, comparando dos pacientes bien 
operados, los cuidados y la prescripción en el perioperatorio pueden hacer la diferencia entre los dos y, en ese sentido, los nuevos cuidados llamados fast track o protocolos multimodales, especialmente documentados en el proyecto ACERTO, pueden acelerar la recuperación del paciente. En la era actual de la medicina basada en evidencia, esos protocolos, probados en estudios aleatorizados y controlados, se han mostrado seguros y superiores a los cuidados tradicionales.

\section{Agradecimientos}

Departamento de Clínica Cirúrgica da UFMT.

\section{Financiación}

JEA-N y bolsista do CNPq (Conselho Nacional de Desenvolvimento Científico e Tecnológico) JCS.

\section{Conflicto de intereses}

No hay conflicto de intereses.

\section{Declaración de autoría}

Todos los autores revisaron el artículo y validaron su versión final.

\section{Referencias bibliográficas}

1. Polk HC Jr, Birkmeyer J, Hunt DR, Jones RS, Whittermore $\mathrm{AD}$, Barraclough B. Quality and Safety in Surgical Care. Ann Surg. 2006; 243(4):439-48.

2. Schifftner TL, Grunwald GK, Henderson WG, Main D, Khuri SF. Relationship of processes and structures of care in general surgery to postoperative outcomes: a hierarchical analysis. J Am Coll Surg. 2007; 204(6):1166-77.

3. Kehlet H, Wilmore DW. Multimodal strategies to improve surgical outcome. Am J Surg. 2002; 183(6):630-41.

4. Fearon KC, Ljungqvist $\mathrm{O}$, von Meyenfeldt $\mathrm{M}$, Revhaug A, Dejong $\mathrm{CH}$, Lassen $\mathrm{K}$, et al. Enhanced recovery after surgery. a consensus review of clinical care for patients undergoing colonic resection. Clin Nutr. 2005; 24(3):466-77.

5. Wind J, Polle SW, Fung Kon Jin PH, Dejong $\mathrm{CH}$, von Meyenfeldt MF, Ubbink DT, et al. Systematic review of enhanced recovery programmes in colonic surgery. Br J Surg. 2006; 93(7):800-9.

6. Khoo CK, Vickery CJ, Forsyth N, Vinall NS, Eyre-Brook IA. A prospective randomized controlled trial of multimodal perioperative management protocol in patients undergoing elective colorectal resection for cancer. Ann Surg. 2007; 245(6):867-72.
7. Rede Interagencial de Informações para a Saúde (RIPSA). Indicadores de recursos [Internet]: IDB, Brasil; 2007 Fecha de consulta: 15 de outubro de 2018]. Disponible en: http:// tabnet.datasus.gov.br/cgi/tabcgi.exe?idb2007/e11.def.

8. de-Aguilar-Nascimento JE, Bicudo-Salomão A, Caporossi C, Silva RM, Cardoso EA, Pádua Santos T. Volume de fluído intravenoso e alta hospitalar precoce em colecistectomia aberta. Rev Col Bras Cir. 2007; 34(6):381-4.

9. de-Aguilar-Nascimento JE, Bicudo Salomão A, Waitzberg DL, Dock-Nascimento DB, Correa MITD, Campos ACL, et al. Diretriz ACERTO de intervenções nutricionais no perioperatório em cirurgia geral eletiva. Rev Col Bras Cir. 2017; 44(6):633-48.

10. Warner MA. Is pulmonary aspiration still an import problem in anesthesia? Current Opin Anaesthesiol. 2000;13(2):215-8.

11. Aguilar-Nascimento JE, de Almeida Dias AL, DockNascimento DB, Correia TDMI, Campos ACL, Portari-Filho $\mathrm{PE}$, et al. Actual preoperative fasting time in Brazilian hospitals: the BIGFAST multicenter study. Ther Clin Risk Manag. 2014; 10:107-12.

12. de-Aguilar-Nascimento JE, Bicudo-Salomão A, Caporossi C, de-Melo Silva R, Cardoso EA, Pádua Santos T. Acerto pósoperatório: avaliação dos resultados da implantação de um protocolo multidisciplinar de cuidados peri-operatórios em cirurgia geral. Rev Col Bras Cir. 2006; 33(3):181-8.

13. Cahill GF Jr. Starvation in man. N Engl J Med. 1970; 282(12):668-75.

14. Faria MS, de Aguilar-Nascimento JE, Pimenta OS, Alvarenga LC Jr, Dock-Nascimento DB, Slhessarenko N. Preoperative fasting of 2 hours minimizes insulin resistance and organic response to trauma after video-cholecystectomy: a randomized, controlled, clinical trial. World J Surg. 2009; 33(6):1158-64.

15. Davisson Correia MIT, Gomes da Silva R. Paradigmas e evidências da nutrição peri-operatória. Rev Col Bras Cir. 2005;32(6):342-7.

16. Brady M, Kinn S, Stuart P. Preoperative fasting for adults to prevent perioperative complications. Cochrane Database Syst Rev. 2003; (4):CD004423.

17. Lobo DN, Hendry PO, Rodrigues G, Marciani L, Totman JJ, Wright JW, et al. Gastric emptying of three liquid oral preoperative metabolic preconditioning regimens measured by magnetic resonance imaging in healthy adult volunteers: a randomised double-blind, crossover study. Clin Nutr. 2009; 28(6):636-41.

18. Perrone F, Aguilar-Nascimento JE, Prado LI, Rabello S, Dock-Nascimento DB. Reducing preoperative fasting with carbohydrates and whey protein reduces the inflammatory response after elective operations. A randomized trial. Clin Nutr Supplements. 2010; 5(2):196-7.

19. Awad S, Blackshaw Pe, Wright JW, Macdonald IA, Perkins AC, Lobo DN. A randomized crossover study of the effects of glutamine and lipid on the gastric emptying time of a preoperative carbohydrate drink. Clin Nutr. 2010; 30(2):165-71. 
20. Brandstrup B, Tønnesen H, Beier-Holgersen R, Hjortsø E, Ørding $\mathrm{H}$, Lindorff-Larsen $\mathrm{K}$, et al. Effects of intravenous fluid restriction on postoperative complications: comparison of two perioperative fluid regimens: a randomized assessorblinded multicenter trial. Ann Surg. 2003;238(5):641-8.

21. Powell-Tuck J, Gosling P, Lobo DN, Allison SP, Carlson GL, Gore M, et al GIFTASUP (British Consensus Guidelines on Intravenous Fluid Therapy for Adult Surgical Patients) [Internet]. Londres: NHS National Library of Health [Fecha de consulta: 8 de abril de 2019]. Disponible en: https://www. bapen.org.uk/pdfs/bapen_pubs/giftasup.pdf

22. Stewart BT, Woods RJ, Collopy BT, Fink RJ, Mackay JR, Keck JO. Early feeding after elective open colorectal resections: a prospective randomized trial. Aust N Z J Surg. 1998; 68(2):125-8.

23. de-Aguilar-Nascimento JE, Göelzer J. [Early feeding after intestinal anastomoses: risks or benefits?] Rev Assoc Med Bras. 2002; 48(4):348-52.

24. Lassen K, Dejong CH, Ljunggvist O, Fearon K, Andersen J, Hannemann $\mathrm{P}$, et al. Nutrional support and oral intake after gastric resection in five northern European countries. Dig Surg. 2005; 22(5):346-52.

25. Lewis SJ, Egger M, Sylvester PA, Thomas S. Early enteral feeding versus "nil by mouth" after gastrointestinal surgery: systematic review and meta-analysis of controlled trials. BMJ. 2001; 323(7316):773-6.

26. Senkal M, Mumme A, Eickhoff U, Geier B, Wulfert D, Joosten $\mathrm{D}$, et al. Early postoperative enteral immunonutrition: clinical outcome and cost-comparison analysis in surgical patients. Crit Care Med. 1997; 25(9):1489-96.

27. Behm B, Stollman N. Postoperative ileus: etiologies and interventions. Clin Gastroenterol Hepatol. 2003;1(2):71-80.

28. Nichols RL, Smith JW, Garcia RY, Waterman RS, Holmes JW. Current practices of preoperative bowel preparation among North American colorectal surgeons. Clin Infect Dis. 1997;24(4):609-19.

29. Peppas G, Alexiou VG, Falagas ME. Bowel cleansing before bowel surgery: major discordance between evidence and practice. J Gastrointest Surg. 2008; 12(5):919-20.

30. Drummond RJ, McKenna RM, Wright DM. Current practice in bowel preparation for colorectal surgery: a survey of the members of the Association of Coloproctology of GB \& Ireland. Colorectal Dis. 2011;13(6):708-10.

31. Wille-Jørgensen P, Guenaga KF, Castro AA, Matos D. Clinical value of preoperative mechanical bowel cleansing in elective colorectal surgery: a systematic review. Dis Colon Rectum. 2003; 46(8):1013-20.

32. Slim K, Vicaut E, Panis Y, Chipponi J. Meta-analysis of randomized clinical trials of colorectal surgery with or without mechanical bowel. Br J Surg. 2004; 91(9):1125-30.

33. Bucher P, Mermillod B, Gervaz P, Morel P. Mechanical bowel preparation for elective colorectal surgery: a meta-analysis. Arch Surg. 2004;139(12):1359-64.

34. Guenaga KF, Matos D, Castro AA, Atallah AN, Wille-Jørgensen P. Mechanical bowel preparation for elective colorectal surgery. Cochrane Database Syst Rev. 2003; (2):CD001544. 